\title{
Metastable bound state of a pair of two-dimensional spatially separated electrons in anti-parallel magnetic fields
}

\author{
S. I. Shevchenko $\dagger$ and E. D. Vol \\ B. I. Verkin Institute for Low Temperature Physics and Engineering National \\ Academy of Sciences of Ukraine, Lenin av. 47 Kharkov 61103, Ukraine
}

\begin{abstract}
We propose a new mechanism for binding of two equally charged carriers in a double-layer system subjected by a magnetic field of a special form. A field configuration for which the magnetic fields in adjacent layers are equal in magnitude and opposite in direction is considered. In such a field an additional integral of motion - the momentum of the pair $\overrightarrow{\mathcal{P}}$ arises. For the case when in one layer the carrier is in the zero $(n=0)$ Landau level while in the other layer - in the first $(n=1)$ Landau level the dependence of the energy of the pair on its momentum $E(\mathcal{P})$ is found. This dependence turns out to be nonmonotonic one : a local maximum and a local minimum appears, indicating the emergence of a metastable bound state of two carrier with the same sign of electrical charge.

PACS numbers: 73.21.-b
\end{abstract}

\section{Introduction}

During last ten years a possibility to measure the effects caused by an interaction of spatially separated carriers in low-dimensional systems has been demonstrated in a number of experiments. An undoubted evidence for such effects was obtained in drag experiments in which a voltage in one conducting layer caused by an electric current in the adjacent layer (separated from the first one by a dielectric layer) was observed. The drag effects have been registered in bilayer systems with the conductivity of the same type in both layers (for instance, the electron-types) [1, 2, 3] and in the layers with the conductivity of the opposite types (electron-type in one layer and the hole-type in the other one) [4, 5]. In the last case the interaction between spatially separated electrons and holes may result not only in the drag effect but also in an electron-hole pairing. The electron-hole pairs may condense into a specific superfluid state in which a supercurrent in one layer is accompanied by a supercurrent in the adjacent layer and these currents have the same absolute value but the opposite directions [6, 17.

The most favorable conditions for the electron-hole pairing are achieved in a case when a strong (quantizing) perpendicular to the layers magnetic field is applied to a † shevchenko@ilt.kharkov.ua 
bilayer electron-hole [8, 9, 10] or electron-electron system [11, 12] (with the total filling factor $\nu_{T}=1$ in the last case). The experimental discovery of the superfluidity of the pairs in such systems has been already reported [13, 14].

The possibility of pairing of spatially separated electrons and holes looks quite natural since there are Coulomb attraction forces between an electron and a hole. Unexpected and less obvious phenomenon consists in that in a strong magnetic field the Coulomb repulsion between spatially separated equally charged carriers may result in a formation of metastable bound pairs. Such an effect takes place in a situation when the magnetic fields applied to the first and the second layer of bilayer electron (or hole) system are antiparallel to each other. This possibility was predicted in our paper [15], where we assume that together with the antiparallel perpendicular to the layers magnetic fields the antiparallel to each other and parallel to the conducting layers electric fields are applied to the system. The disadvantage of the situation considered in 15. is that the presence of the electric fields may result in an instability of the system with a macroscopic number of the pairs.

In this paper we show that a formation of a metastable bound state of spatially separated electrons (or holes) can emerge without the electric fields applied to the system and formulate the conditions of appearance of such a bound state.

\section{Electron-electron pair in antiparallel magnetic fields}

Let us consider two two-dimensional electron layers with the interlayer distance $d$ embedded in a dielectric matrix with the dielectric constant $\varepsilon_{0}$. Let the magnetic field in the top layer (layer 1$)$ is $\mathbf{B}_{1}=(0,0,-B)$ and the magnetic field in the bottom layer (layer 2 ) is $\mathbf{B}_{2}=(0,0, B)$ (the $z$ axis is chosen perpendicular to the layers). The possible way of realization of such a configuration of magnetic fields will be discussed in the end of the paper. We specify the case when there is one electron belonging to the zero Landau level in the layer 1 and one electron belonging to the first Landau

level in the layer 2 . In the symmetric gauge the vector potential in the layer 1 is equal to $\mathbf{A}_{1}\left(\mathbf{r}_{1}\right)=(B y / 2,-B x / 2,0)$ and in the layer $2 \mathbf{A}_{2}\left(\mathbf{r}_{2}\right)=(-B y / 2, B x / 2,0)$. The Hamiltonian of a pair of interacting electrons has the form

$$
H=H_{1}+H_{2}+V_{C}\left(\left|\mathbf{r}_{1}-\mathbf{r}_{2}\right|\right)
$$

where

$$
\begin{aligned}
& H_{1}=\frac{\left(\hat{p}_{1 x}+\frac{e B}{2 c} y_{1}\right)^{2}}{2 m_{1}}+\frac{\left(\hat{p}_{1 y}-\frac{e B}{2 c} x_{1}\right)^{2}}{2 m_{1}}, \\
& H_{2}=\frac{\left(\hat{p}_{2 x}-\frac{e B}{2 c} y_{2}\right)^{2}}{2 m_{2}}+\frac{\left(\hat{p}_{2 y}+\frac{e B}{2 c} x_{2}\right)^{2}}{2 m_{2}}, \\
& V_{C}=\frac{e^{2}}{\varepsilon_{0}\left|\mathbf{r}_{1}-\mathbf{r}_{2}\right|}=\frac{e^{2}}{\varepsilon_{0} \sqrt{\left(x_{1}-x_{2}\right)^{2}+\left(y_{1}-y_{2}\right)^{2}+d^{2}}} .
\end{aligned}
$$

Here $\mathbf{r}_{1}, \mathbf{r}_{2}$ are the two-dimensional vectors. We set the electron charge equals to $-e$. 
In a strong magnetic field the Bohr radiuses of the electrons $a_{B}^{(1)}=\varepsilon_{0} \hbar^{2} / m_{1} e^{2}$, $a_{B}^{(2)}=\varepsilon_{0} \hbar^{2} / m_{2} e^{2}$ can be much larger then the magnetic length $\ell_{0}=(c \hbar / e B)^{1 / 2}$. In this case the Coulomb interaction can be taken into account as a perturbation. It is known that the quantum problem of a particle in a quantizing magnetic field has a large degeneracy (in the symmetric gauge with respect to the quantum number $m$, the $z$ projection of the angular momentum). Therefore, the common formulation of the theory of perturbations should be based on a solution of a secular equation. But such an approach is not an optimal method for the study of this problem. Here we use another approach based on the projection of the Hamiltonian (11) into the subspace of the states of the pair of electrons in which the electrons in the layer 1 and 2 are frozen on the zero and the first Landau levels, correspondingly (it is just the approach used in the theory of the quantum Hall effect). Then the kinetic energy operator for the electron in the layer 1

$$
H_{1}=\frac{\left(\Pi_{1 x}\right)^{2}+\left(\Pi_{1 y}\right)^{2}}{2 m_{1}}=\hbar \omega_{1}\left(a_{1}^{+} a_{1}+\frac{1}{2}\right)
$$

is projected to $\bar{H}_{1}=\hbar \omega_{1} / 2$ (here and further the bar symbols indicate the projected operators) which is constant. We will omit this constant in the further consideration. In (15) $\omega_{1}=e B / m_{1} c$ is the cyclotron frequency for the electron in the layer 1, $\Pi_{1 x} \equiv p_{1 x}+\frac{e B}{2 c} y_{1}, \Pi_{1 y} \equiv p_{1 y}-\frac{e B}{2 c} x_{1}$, the electron kinematic momentum components, $a_{1}^{+}=\frac{\ell_{0}}{\hbar \sqrt{2}}\left(\Pi_{1 x}-i \Pi_{1 y}\right), a_{1}=\frac{\ell_{0}}{\hbar \sqrt{2}}\left(\Pi_{1 x}+i \Pi_{1 y}\right)$, the creation and the annihilation operators for the electron 1 on a zero Landau level. It follows from the commutation relation for $\Pi_{i}^{1}\left(\left[\Pi_{1 x}, \Pi_{1 y}\right]=i \hbar^{2} / \ell_{0}^{2}\right)$ that $\left[a, a^{+}\right]=1$ as it should be. Analogously, for the electron in the layer 2 one can find

$$
H_{2}=\frac{\Pi_{2 x}^{2}+\Pi_{2 y}^{2}}{2 m_{2}}=\hbar \omega_{2}\left(a_{2}^{+} a_{2}+\frac{1}{2}\right)
$$

and $\bar{H}_{2}=3 \hbar \omega_{2} / 2$.

To project out the $V_{C}\left(\left|\mathbf{r}_{1}-\mathbf{r}_{2}\right|\right)$ operator it is convenient to rewrite it in a Fourierrepresentation form (we follow the procedure [16])

$$
V_{C}=\frac{e^{2}}{2 \pi \varepsilon_{0}} \int d^{2} k \frac{\exp (-k|d|)}{|k|} \exp \left(i k_{x}\left(x_{1}-x_{2}\right)+i k_{y}\left(y_{1}-y_{2}\right)\right),
$$

where $|k|=\sqrt{k_{x}^{2}+k_{y}^{2}}$.

The coordinates of the electron in the layer 1 can be presented as

$$
x_{1}=X_{1}-\frac{\ell_{0}^{2}}{\hbar} \Pi_{1 y}, \quad y_{1}=Y_{1}+\frac{\ell_{0}^{2}}{\hbar} \Pi_{1 x}
$$

where $X_{1}, Y_{1}$ are the coordinates of the centers of its orbit in the magnetic field. The operators $X_{1}$ and $Y_{1}$ satisfy the following commutation relations: $\left[X_{1}, Y_{1}\right]=-i \ell_{0}^{2}$. Besides that, $X_{1}$ and $Y_{1}$ commute with the momenta components $\Pi_{1 x}$ and $\Pi_{1 y}$. Analogously, for the electron in the layer 2 we have

$$
x_{2}=X_{2}+\frac{\ell_{0}^{2}}{\hbar} \Pi_{2 y}, \quad y_{2}=Y_{2}-\frac{\ell_{0}^{2}}{\hbar} \Pi_{2 x}
$$


where $\left[X_{2}, Y_{2}\right]=i \ell_{0}^{2}$. Now the projection of the operator $V_{C}$ is reduced to the independent projection of two commuting operators $U_{1}$ and $U_{2}$ :

$$
\begin{aligned}
& \hat{U}_{1} \equiv \exp \left(-i k_{x} \frac{\ell_{0}}{\hbar} \Pi_{1 y}+i k_{y} \frac{\ell_{0}}{\hbar} \Pi_{1 x}\right)=\exp \left[\frac{\ell_{0}}{\sqrt{2}}\left(k a_{1}^{+}-\bar{k} a_{1}\right)\right], \\
& \hat{U}_{2} \equiv \exp \left(i k_{x} \frac{\ell_{0}}{\hbar} \Pi_{2 y}-i k_{y} \frac{\ell_{0}}{\hbar} \Pi_{2 x}\right)=\exp \left[\frac{\ell_{0}}{\sqrt{2}}\left(\bar{k} a_{2}^{+}-k a_{2}\right)\right],
\end{aligned}
$$

where the notation $k \equiv k_{x}+i k_{y}$ is used. The projection of these operators can be easily done:

$$
\bar{U}_{1}=\left\langle 0\left|\hat{U}_{1}\right| 0\right\rangle=\exp \left(-\frac{|k|^{2} \ell_{0}^{2}}{4}\right), \quad \bar{U}_{2}=\left\langle 1\left|\hat{U}_{2}\right| 1\right\rangle=\exp \left(-\frac{|k|^{2} \ell_{0}^{2}}{4}\right)\left[1-\frac{|k|^{2} \ell_{0}^{2}}{2}\right] .
$$

Substituting Eq. (12) into Eq. (17) we arrive to the following expression for the operator $\bar{V}_{C}$

$$
\bar{V}_{C}=\frac{e^{2}}{2 \pi \varepsilon_{0}} \int d^{2} k \frac{e^{-|k| d}}{|k|} e^{-\frac{|k|^{2} \ell_{0}^{2}}{2}}\left[1-\frac{|k|^{2} \ell_{0}^{2}}{2}\right] e^{i k_{x}\left(X_{1}-X_{2}\right)+i k_{y}\left(Y_{1}-Y_{2}\right)} .
$$

Since the operators $X_{1}-X_{2}$ and $Y_{1}-Y_{2}$ commute with each other and with the Hamiltonian (11) these operators are the integrals of motion. The appearance of the integrals of motions in the problem considered is not accidental. The point is that the Hamiltonian (II) and the Hamiltonian of the electron-hole pair coincide with each other up to a sign of the Coulomb interaction. In the last (electron-hole) case there is the integral of motion - the momentum of the pair $\overrightarrow{\mathcal{P}}[17]$

$$
\overrightarrow{\mathcal{P}}=\left(-i \hbar \frac{\partial}{\partial \mathbf{r}_{1}}+\frac{e}{c} \mathbf{A}_{1}\right)+\left(-i \hbar \frac{\partial}{\partial \mathbf{r}_{2}}-\frac{e}{c} \mathbf{A}_{2}\right)-\frac{e}{c}\left[\mathbf{B} \times\left(\mathbf{r}_{1}-\mathbf{r}_{2}\right)\right] .
$$

In our problem the momentum of the pair $\overrightarrow{\mathcal{P}}$ is also the integral of motion. Comparing Eq. (14) with Eqs. (8) and (9) we find the relation between the components of the momentum $\overrightarrow{\mathcal{P}}$ and the operators $X_{1}-X_{2}$ and $Y_{1}-Y_{2}$ :

$\mathcal{P}_{x}=\frac{\hbar}{\ell_{0}^{2}}\left(Y_{2}-Y_{1}\right) \quad$ and $\quad \mathcal{P}_{y}=-\frac{\hbar}{\ell_{0}^{2}}\left(X_{2}-X_{1}\right) \quad$ or $\quad \overrightarrow{\mathcal{P}}=\frac{\hbar}{\ell_{0}^{2}}\left(\mathbf{R}_{2}-\mathbf{R}_{1}\right) \times e_{z}$,

where $e_{z}$ is the unit vector in $z$-direction. Taking Eq. (15) into account we rewrite the energy of the electron pair as the function of its momentum $\overrightarrow{\mathcal{P}}$ :

$$
\Delta E_{01}(\overrightarrow{\mathcal{P}})=\frac{e^{2}}{2 \pi \varepsilon_{0}} \int d^{2} k \frac{e^{-|k| d}}{|k|} e^{\frac{i k_{x} \ell_{0}^{2}}{2} \mathcal{P}_{y}-\frac{i k_{y} \ell_{0}^{2}}{2}} \mathcal{P}_{x} e^{-\frac{k^{2} \ell_{0}^{2}}{2}}\left(1-\frac{k^{2} \ell_{0}^{2}}{2}\right) .
$$

Prior to analyze Eq. (16) we note the following.

1. Using the method presented here one can also find the dependence of the energy of the pair on its momentum $\overrightarrow{\mathcal{P}}$ in a general case, when the electron in the layer 1 is "frozen" on the $n_{1}$-th Landau level and the electron in the layer 2 - on the $n_{2}$-th level. The final result is

$\Delta E_{n_{1} n_{2}}(\overrightarrow{\mathcal{P}})=\frac{e^{2}}{2 \pi \varepsilon_{0}} \int d^{2} k \frac{e^{-|k| d}}{|k|} e^{\frac{i k_{x} \ell_{0}^{2}}{2}} \mathcal{P}_{y}-\frac{i k_{y} \ell_{0}^{2}}{2} \mathcal{P}_{x} e^{-\frac{k^{2} \ell_{0}^{2}}{2}} L_{n_{1}}\left(\frac{k^{2} \ell_{0}^{2}}{2}\right) L_{n_{2}}\left(\frac{k^{2} \ell_{0}^{2}}{2}\right)$,

where $L_{n}(x)$ are the Laguerre polynomials. 
2. The variant of the theory of perturbations used here allows to solve the problem also in the case when the condition $a_{B} \gg \ell_{0}$ is satisfied only for the one particle in the pair, while the Bohr radius for the other particle can be of order $\ell_{0}$. Such a situation may take place if the effective masses of the carries differ considerably from each other $\left(m_{1} \ll m_{2}\right)$, see [15].

Returning to the analysis of the result (16) we consider only the simplest case $d \rightarrow 0$. The results obtained below remain qualitatively correct if $d \lesssim \ell_{0}$. At $d=0$ the integral in the r.h.s. of Eq. (16) is calculated analytically. The result is

$$
\Delta E_{01}(p)=\frac{e^{2}}{\varepsilon \ell_{0}} \sqrt{\frac{\pi}{2}} \frac{1}{2}\left[\left(1-\frac{p^{2}}{2}\right) \mathrm{I}_{0}\left(\frac{p^{2}}{4}\right)-\frac{p^{2}}{2} \mathrm{I}_{1}\left(\frac{p^{2}}{4}\right)\right] e^{-\frac{p^{2}}{4}},
$$

where $p=\mathcal{P} \ell_{0} / \hbar$ is the dimensionless momentum of the pair, $\mathrm{I}_{0}(x)$ and $\mathrm{I}_{1}(x)$ are the modified Bessel functions of the zero and the first order, correspondingly. Using the asymptotic expressions for $\mathrm{I}_{0}(x)$ and $\mathrm{I}_{1}(x)$ one can find from Eq. (18) the dependence $\Delta E_{01}(p) \equiv \epsilon(p)$ at small and large $p$.

1) At $p \ll 1$

$$
\epsilon(p) \cong E_{0}+\frac{\hbar^{2} p^{2}}{2 M_{*} \ell_{0}},
$$

where the energy $E_{0}$ and the effective mass of the pair $M_{*}$ read as

$$
E_{0}=\frac{e^{2}}{2 \varepsilon_{0} \ell_{0}} \sqrt{\frac{\pi}{2}}, \quad \text { and } \quad M_{*}=\left(\frac{2}{\pi}\right)^{1 / 2} \frac{4 \varepsilon_{0} \hbar^{2}}{e^{2} \ell_{0}} .
$$

One should note that in the approximation used the effective mass $M_{*}$ is determined only by the interaction between electrons. The bare masses $m_{1}$ and $m_{2}$ do not enter into the expression for $M_{*}$. The bare masses determine only the Larmour frequencies $\omega_{1}$ and $\omega_{2}$.

2) At $p \gg 1$

$$
\epsilon(p) \simeq \frac{4}{\sqrt{2 \pi}} \frac{E_{0}}{p}
$$

As it follows from Eq. (20) and (21) the energy of the electron pair as the function of the momentum $p$ increases at small momenta and decreases at large $p$. Numerical estimates show that the function $\epsilon(p)$ reaches its maximum $\epsilon_{m}=1.148 E_{0}$ at $p=p_{m}=1.194$. The dependence $\epsilon(p)$ is shown in Fig.1.

\section{Discussion}

The existence of the local minimum of $\epsilon(p)$ at $p=0$ is the main result. It has important consequences. There are simple and transparent reasons for the appearance of the local minimum in the case when the electron in one layer belongs to the zero Landau level and the electron in the adjacent layer - to the first Landau level. The wave functions are shown schematically in Fig. 2 (rigorously speaking, the electron density distributions in the layers are presented in this figure). 


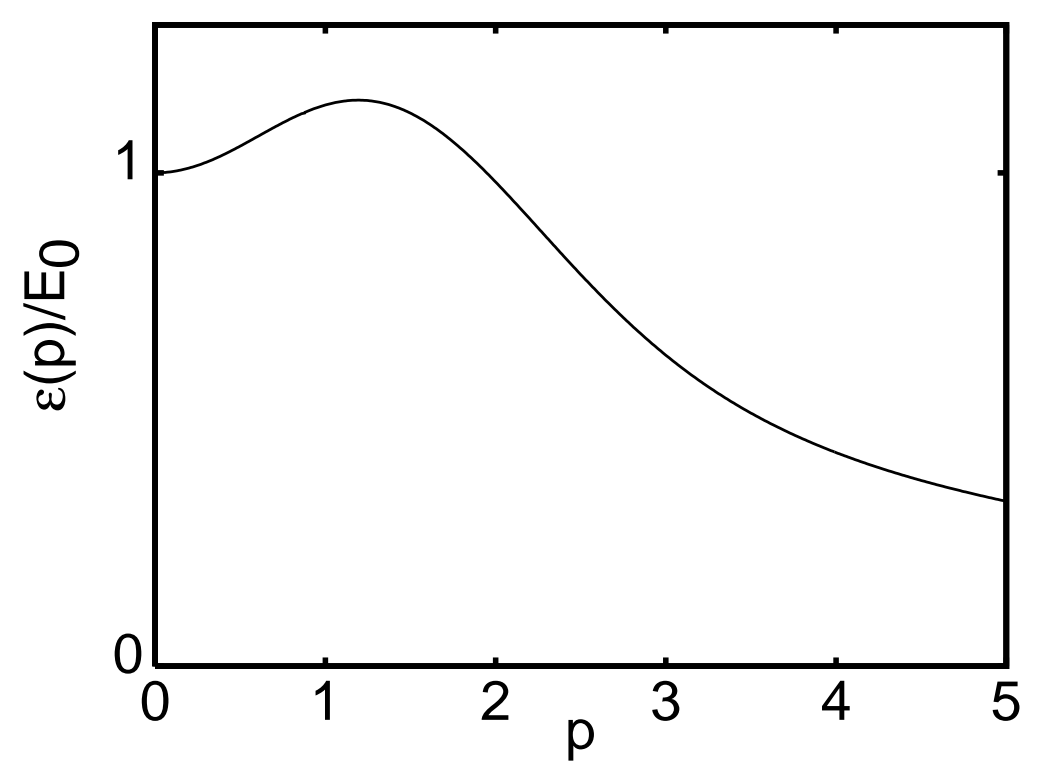

Figure 1. The dependence of the energy of the electron pair on its momentum.

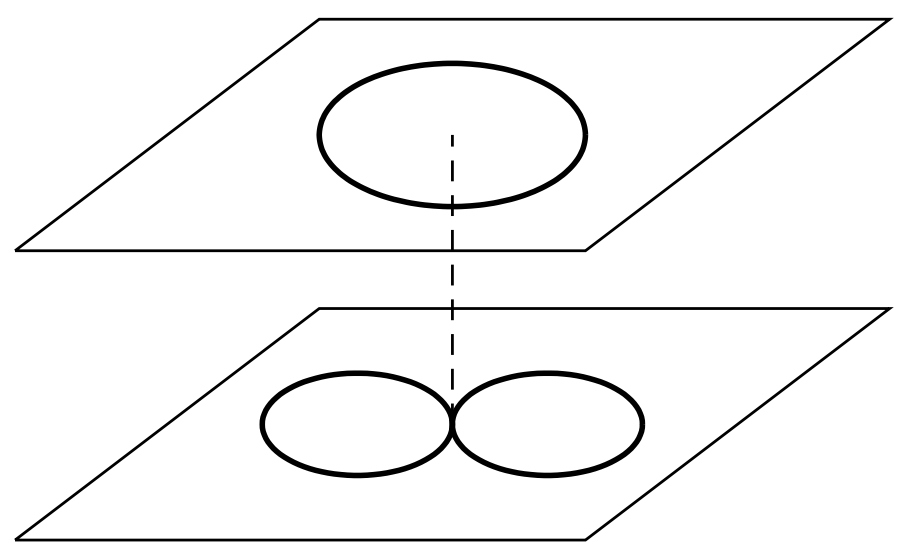

Figure 2. Configuration of the electron density in two layers in the $p=0$ pair state. 
It is physically evident that in the case when the centers of the orbits of both electron coincide $(p=0)$ the energy of the Coulomb repulsion is smaller (local minimum) than at small displacements from this configuration. One should note that the interaction energy $\epsilon(p)$ is positive at all $p$, which is natural since the repulsion forces act between the electrons. But the presence of the minimum gives an evidence of a formation of a metastable bound state of two electrons from the adjacent layers. Actually, it is easy to find the average distance between the electron of the pair with the momentum $\overrightarrow{\mathcal{P}}$

$$
r \equiv\left(\left\langle\psi\left|\left(r_{1}-r_{2}\right)^{2}\right| \psi\right\rangle\right)^{1 / 2}=\ell_{0}\left(4+p^{2}\right),
$$

where $|\psi\rangle$ is the wave function of the pair with the momentum $\overrightarrow{\mathcal{P}}$. Therefore, these is a one to one correspondence between the momentum and the size of the pair. If the pair has the momentum $\mathbf{p}=0$ then the average distance is $r=2 \ell_{0}$. To separate the electrons on the infinite distance from each other (when $\epsilon(p=\infty)=0$ ) the energy barrier $\epsilon\left(p_{m}\right)-\epsilon(0)=0.148 E_{0}$ should be overcome. It means that the electron pairs with small momenta $p$ are in the quasibound state stable against different scattering processes in which the shift of the momentum of the pair is smaller then $p_{m}$. The bound electron pairs are the bosons and, therefore, in the system of such pairs one can expect the transition into the superfluid state if the density of the pair is quite large. Since the pairs are charged this state should be superconductive one. Strictly speaking, the problem of a transition of the electron pairs into the superfluid state in the system studied requires further analysis. Since the bound state of the pairs does not correspond to the true minimum of the energy the thermodynamic arguments cannot be used to establish the conditions of the existence of the superfluid state. To clarify this question the probability of the transition from the state with a given number of the pair with zero or small momenta should be found. We expect that due to the presence of the barrier for pair decoupling in the two-particle problem a barrier for the destruction of the coherent state will exist in the many-particle problem as well. Therefore, at the temperature smaller then the value of the barrier the time of life of the coherent state will be large.

In conclusion, we discuss shortly the question about the possibility to realize the magnetic field configuration required. This question is not so simple from the experimental point of view. Actually, the fields should be of order of $1 \div 10 \mathrm{~T}$. But we think this obstacle can be overcome. We propose two ways of possible solution of this problem. First, the required (antiparallel) configuration of the magnetic fields can be realized using the magnetized stripes of magnetically-hard materials (like Dy) deposited on the bilayer structure. In Ref. [18 such a method was used for designing periodic magnetic fields with $B_{\max }=1 \mathrm{~T}$ with the aim to study the conducting properties of a two-dimensional electron gas in such fields. Another possible way to create antiparallel magnetic field configuration can be based on using antiferromagnetic systems in which the spins in each layer are ferromagnetically ordered while they are directed antiparallel in adjacent layers. For example, such properties demonstrate the layered manganites $(\mathrm{LaSr})_{n+1} \mathrm{Mn}_{n} \mathrm{O}_{3 n+1}$, the compounds widely studied now (see, for instance, review [19]). 
This work is supported by the INTAS grant No 01-2344.

[1] Gramila T J, Eisenstein J P, MacDonald A H, Pfeiffer L N and West K W 1991 Phys. Rev. Lett. 661261.

[2] Gramila T J, Eisenstein J P, MacDonald A H, Pfeiffer L N and West K W 1993 Phys. Rev. B 47 12957.

[3] Rubel H, Fisher A, Dietsche W D, von Klitzing K and Eberl K 1997 Phys. Rev. Lett. 781763.

[4] Sivan U, Solomon P M and Shtrikman H 1992 Phys. Rev. Lett. 681196.

[5] Rubel H, Fisher A, Dietsche W D, Jorger C, von Klitzing K and Eberl K 1998 Physica B 249-251 859.

[6] Lozovik Yu E and Yudson V I, 1976 Zh. Eksp. Teor. Fiz. 71738 [1976 Sov. Phys. JETP 44 389].

[7] Shevchenko S I 1976 Fiz. Nizk. Temp. 2505 [1976 Sov. J. Low Temp. Phys. 2 251].

[8] Kuramoto Y and Horie C 1978 Solid St. Comm. 25713.

[9] Lerner I V and Lozovik Yu E 1981 Zh. Eksp. Teor. Fiz. 80 1488[1981 Sov. Phys. JETP 53 763].

[10] Shevchenko S I 1998 Phys. Rev. B 5714809.

[11] Wen X G and Zee A 1992 Phys. Rev. Lett. 691811.

[12] Moon K, Mori H, Yang K, Girvin S N, MacDonald, Zheng L, Yoshioka D and Zhang S C 1995 Phys. Rev B $\mathbf{5 1} 5138$

[13] Spielman I B, Eisenstein J P, Pfeiffer L N and West K W 2001 Phys. Rev. Lett. 87036803.

[14] Kellog M, Spielman I B, Eisenstein J P, Pfeiffer L N and West K W 2002 Phys. Rev. Lett. 88 126804.

[15] Vol E D and Shevchenko S I 2001 Fiz. Nizk. Temp. 271376 [2001 Low Temp. Phys. 27 1014].

[16] Izava Z F 1997 Phys. Rev. B 557771.

[17] Gor'kov L P and Dzyaloshinslii 1967 Zh. Eksp. Teor. Fiz. 53717 [1968 Sov. Phys. JETP 26 449].

[18] Ye P D, Wiess D, Gerhadts R R, Seeger M, von Klitzing K, Eberl K and Nickel H 1995 Phys. Rev. Lett. 743013.

[19] Kagan M Yu and Kugel K.I. 2001 Physics-Uspekhi 44553. 